\title{
Antipsicologismo y platonismo en el siglo XIX: Herbart, Bolzano y Lotze
}

\section{(Antipsychologism and Platonism in the 19th Century: Herbart, Bolzano and Lotze)}

\author{
Luis NIEL
}

Recibido: 4 de marzo de 2013

Aceptado: 2 de julio de 2013

\section{Resumen}

El presente artículo analiza la obra de tres pensadores del siglo XIX: Herbart, Bolzano y Lotze. Más allá de sus diferencias, presentaremos dos rasgos esenciales comunes: su rechazo al psicologismo en tanto radicalización de la psicología como disciplina fundacional última (antipsicologismo) y la idea de una 'realidad' ideal independiente tanto de lo sensible como de lo mental y lo lingüístico, que sustenta dicha posición (platonismo). A modo de conclusión, mostraremos cómo, según estos autores, dicho trasfondo ideal platónico es lo que da sustento a todo pensar con sentido.

Palabras clave: Antipsicologismo, platonismo, siglo XIX, Herbart, Bolzano, Lotze.

\begin{abstract}
The article addresses the works of three 19th-century philosophers: Herbart, Bolzano and Lotze. Despite their differences, I will analyze two essential features they share: their refusal to psychologism as the radicalization of psychology as the ultimate source of philosophy (antipsychologism), and the positing of an ideal 'reality', independent from both sensibility and the mental and linguistic dimensions, upon which the refusal to psychologism is founded (Platonism). As a conclusion, I will show how, according to these authors, this platonic, ideal background constitutes the very foundation of every meaningful thought.
\end{abstract}

Keywords: Antipsychologism, platonism, 19th century, Herbart, Bolzano, Lotze. 
Es casi un lugar común situar históricamente a Edmund Husserl y a Gottlob Frege como los 'padres' del antipsicologismo, es decir, del rechazo y de la refutación de aquellas corrientes decimonónicas que pretendían fundar la lógica y la matemática en la psicología. ${ }^{1}$ Dicha caracterización de 'progenitores' del antipsicologismo no es del todo justa, si se tienen en cuenta tres antecedentes importantes en la filosofía del siglo XIX (i.e. anteriores a Frege y a Husserl), los cuales, en mayor o menor medida, dejaron un aporte relevante en el marco del debate con el psicologismo y sostuvieron una clara postura antipsicologista sobre la base de ciertas ideas platónicas; éstos fueron Johann F. Herbart, Bernard Bolzano y Rudolf Hermann Lotze. Estos pensadores constituyen el antecedente histórico más inmediato de Husserl y de Frege en cuanto al problema del psicologismo, en el marco de un mismo ámbito intelectual, i.e. el mundo académico germano-parlante (con esto nos referimos evidentemente tanto a la academia alemana como a la austríaca). ${ }^{2}$

El presente artículo se centra en la obra de Herbart, de Bolzano y de Lotze, haciendo hincapié en los siguientes aspectos: en primer lugar, presentaremos sus posiciones antipsicologistas a partir del contraste con los postulados psicologistas contrapuestos a éstas. En segundo lugar, mostraremos que en los tres casos considerados se puede hallar un trasfondo platónico que sustenta dichas posiciones antipsicologistas. En tercer lugar, analizaremos las implicaciones ontológicas o incluso 'meta-ontológicas' 3 que se presentan a partir de dicho platonismo; esto nos permi-

1 Cf. Frege 1884, p. 37; Frege 1893, p. XIX; Husserl 1900, Cap. 7.

2 Cabe aclarar que no es intención del presente artículo ofrecer una interpretación histórica, ni buscar bases textuales o filológicas que corroboren alguna presunta influencia directa o indirecta de Herbart, Bolzano o Lotze en la obra de Frege o Husserl. Cualquier conexión que pueda entreverse con la obra de éstos será, en cualquier caso, un resultado secundario del presente artículo. La influencia en la obra de Husserl es explícita, como él mismo reconoce (cf. Husserl 1900, Cap. 11; Husserl, E., Ideen zu einer reinen Phänomenologie und phänomenologischen Philosophie. Drittes Buch: Die Phänomenologie und die Fundamente der Wissenschaften, Husserliana V, The Hague, Nijhoff, 1971, pp. 57-58; Husserl 1979, pp. 156-157). El caso de Frege es más complejo, ya que éste nunca se caracterizó por reconocer influencias de otros autores, sino que intentó más bien marcar, ex negativo, sus diferencias con otras posiciones. Si bien hay fundadas dudas en cuanto a la posibilidad de que Frege haya leído a Bolzano (cf. Sundholm, Göran, "When, and Why, did Frege Read Bolzano?", en Th. Childers (ed.), The Logical Yearbook 1999, Philosophia Publishers, Czech Academy of Science, Praga, 2000; cf. Künne, W., Die philosophische Logik Gottlob Freges, Klostermann, Hamburg, 2010, pp. 759-769; cf. Dummett 1996, p. vii), Frege conocía de hecho la obra de Lotze e incluso asistió a un seminario dictado por éste (como Künne sostiene con pruebas concretas en la Introducción de la obra citada anteriormente). Por supuesto, otra cuestión diferente y muy discutible es si hubo o no una influencia de Lotze en el pensamiento de Frege, tema que generó un conocido debate entre Michael Dummett y Hans Sluga (cf. Sluga, H., "Frege and the Rise of Analytic Philosophy", en Inquiry, 18, 1975; Sluga, H., Gottlob Frege, London, Routledge, 1980 (Cap.2); Sluga, H., "Frege's Alleged Realism", en Sluga, H. (ed.), Meaning and Ontology in Frege's Philosophy, New York, Garland, 1993; Dummett 1996, Caps. 5-6).

3 Me refiero a la postulación ciertas 'entidades platónicas' de tipo semántico, que incluso exceden o 'desbordan' el marco de la ontología, como es en el caso de la obra de Bolzano; de allí el uso del entrecomillado. 
tirá ver el alcance y el sentido del mismo. En cualquier caso, nuestra intención será mostrar que dichas cuestiones ontológicas (eventualmente, 'meta-ontológicas') constituyen una problemática filosófica fundamental, aún cuando éstas, en algunos casos, hubiesen sido explícitamente consideradas como secundarias por los mismos pensadores.

Creemos firmemente que un detenido análisis ontológico y semántico sobre la posibilidad real de entidades ideales, independientes tanto de la realidad (sensible) como de las actividades cognitivo-subjetivas, tiene un significado central para cualquier estudio filosófico que se jacte de analizar cuestiones de fondo. Además, consideramos que ciertos aportes del 'realismo semántico o platónico' que analizaremos a continuación, están en condiciones de presentar excelentes argumentos filosóficos contra toda forma de relativismo (psicologismo, neurocientificismo, sociologismo, etc.). Por supuesto, dichas convicciones exceden con creces el delimitado marco histórico-conceptual de nuestro análisis, cuya meta se circunscribe a exponer algunos de los elementos principales del antipsicologismo platónico, que hallamos -cada uno con su motivación, en su contexto y con su grado de profundidad de análisis- en la obra de Herbart, de Bolzano y de Lotze. En cualquier caso, creemos que una buena comprensión del significado de dicho 'realismo platónico' puede aportar firmes herramientas para entender porqué el materialismo en sus diversas formas (naturalista, histórico, dialéctico, etc.), en última instancia, termina siendo una forma de anti-filosofía 4 , al desconocer una esfera de 'realidad' que se resiste a cualquier tipo de reduccionismo.

\section{Introducción: psicologismo, antipsicologismo y platonismo}

¿Qué es el psicologismo? Como lo dice la misma palabra, el 'psicologismo' es una forma de radicalización de la psicología o incluso una "ideología basada en la psicología". 5 Esta tendencia 'ideológica' fue particularmente fuerte durante el XIX. Históricamente, podemos remontarnos a la primera mitad del siglo XIX, momento en el que el idealismo alemán fue perdiendo su prestigio y ganando un paulatino rechazo. Esta situación se vio acompañada por el avance de las ciencias -y el concomitante surgimiento del positivismo-, en particular de la psicología de base empírica, que se presentó en clara contraposición con la psicología racionalista moderna. En este contexto, la psicología experimental tuvo un desarrollo sin precedentes. Si bien es cierto que el neo-kantismo fue una de las escuelas filosóficas más influ-

\footnotetext{
${ }^{4}$ Carl Stumpf, discípulo de Lotze, sostiene que el materialismo es siempre una "corriente no-filosófica (unphilosophische Strömung)" (Stumpf, C., "Die Wiedergeburt der Philosophie", en Stumpf, C., Philosophische Reden und Vorträge, Leipzig, J.A. Barth, 1910, p. 164).

5 Cf. Jacquette 2003, p. 1. Todas las traducciones del presente artículo son mías.
} 
yentes en esta época, ciertas posiciones naturalistas y positivistas ocuparon gradualmente buena parte del terreno que otrora ocupaba la filosofía trascendental de Kant. Dichas posiciones explicaban los procesos cognitivos humanos no ya a partir de las condiciones a priori, sino a partir de investigaciones empíricas, como es el caso del fisiologismo de Hermann von Helmholtz (1821-1894) o del psicologismo de Wihelm Wundt (1832-1920).6

Es muy difícil explicar en pocas palabras de qué trata el psicologismo, puesto que no se puede hablar de una 'escuela psicologista' con una doctrina homogénea, sino más bien de una tendencia generalizada en la filosofía del siglo XIX, que puede observarse en la obra de Wundt, de John Stuart Mill (1803-1873), de Christoph von Sigwart (1830-1904), y de Theodor Lipps (1851-1914), por mencionar sólo algunas de sus figuras más prominentes. ${ }^{7}$ En cualquier caso, fue una posición muy extendida en la filosofía decimonónica. A pesar de su auge y como consecuencia de las duras críticas antipsicologistas que fueron apareciendo paulatinamente, 'psicologismo' pasó a ser un término negativo y peyorativo entre finales del siglo XIX y principios del XX; nos encontramos así con acusaciones de 'psicologista' que se intercambiaban de una corriente filosófica a otra ${ }^{8}$, en muchos casos más con el intento de desacreditar a otros, que atendiendo al auténtico contenido de las diversas posiciones. ${ }^{9}$

Haciendo caso omiso de las diversas formas y variantes, podemos decir que el 'psicologismo' tiene que ver básicamente con la idea de una fundamentación de la lógica y de la matemática a partir de la psicología; es decir, las leyes 10 de la lógica y de la matemática se justifican a partir de los procesos de pensamiento mediante los cuales llegamos a ellas. En este marco se podrían mencionar posiciones que van desde una afirmación de la lógica en tanto mera 'técnica' o 'arte' (Kunstlehre) para educar el pensar correcto, a corrientes nominalistas extremas, que directamente sostienen que los objetos de la lógica no son más que entidades del pensamiento humano. ${ }^{11}$ En general, un elemento común del psicologismo es el negarle a (los objetos de) la lógica un 'estatuto ontológico' propio.

\footnotetext{
${ }^{6}$ Cf. Kusch 1995, p. 2.

7 Para un análisis detallado de las diversas posiciones psicologistas cf. Kusch 1995, en particular el Capítulo 5.

8 Cf. los estudios estadísticos de Kusch 1995, pp. 95-98.

${ }^{9}$ Más allá del antipsicologismo que caracterizó a buena parte de la tradición filosófica del siglo XX, difícilmente pueda decirse que el psicologismo sea cosa del pasado. Dale Jacquette sostiene que, lejos de haber sido extirpado de la filosofía, aún hay argumentos muy plausibles para su defensa, y que dicha empresa es hoy muy productiva para la filosofía (cf. Jacquette 2003, pp. 9-17). El caso más estridente de nuestros tiempos es quizás el de las neurociencias en su afán de posicionarse como una suerte de prima philosophia.

${ }^{10}$ Frege lleva a cabo un análisis muy elocuente del doble sentido del término 'ley': en sentido descriptivo (e.g. la ley de la gravedad) y en sentido prescriptivo (e.g. una ley moral) (cf. Frege 1893, p. xv). ${ }^{11}$ En su época, es quizás Husserl el autor que más claramente se detiene a analizar las diversas posiciones psicologistas y los argumentos en contra de éstas (cf. Husserl 1900).
} 
Ahora, en un sentido positivo, ¿qué es el antipsicologismo? Así como en el caso del psicologismo, aquí también nos encontramos con dificultades similares, puesto que tampoco podemos hablar de una corriente homogénea, sino más bien de una tendencia filosófica que se posiciona claramente en contra de la radicalización de la psicología. No obstante, se puede observar que, más allá de algunas diferencias entre argumentos y posiciones, hay algunos ejes centrales en los que coinciden la mayoría de los antipsicologistas del siglo XIX, como por ejemplo la diferenciación categórica entre lo objetivo (lo pensado) y lo subjetivo (el pensar), y la caracterización de lo 'objetivo', i.e. el objeto de la lógica, en tanto entidad ideal en sentido platónico.

Algunas denominaciones posibles para esta vertiente decimonónica de 'antipsicologismo platónico' -entre las que, por supuesto, debemos incluir a Frege y a Husserl (a este último, por lo menos en el período de las Logische Untersuchungen)- son: 'realismo lógico'12, 'realismo semántico'13, o 'platonismo lógico'. 14 En este marco, no hay que entender 'platonismo' en un sentido histórico estricto, sino, en general, como la posición que sostiene una división entre una realidad cósica, sensible, y una realidad ideal, suprasensible, y que, además, afirma la posibilidad 'real' de esta última. El caso del término 'realismo' es más complejo, puesto que es un concepto que da lugar a numerosas ambigüedades (y de hecho las ha dado a lo largo de la historia de la filosofía). 15 Indudablemente, en este contexto el término 'realismo' tiene un sentido lógico-semántico, que hace referencia a una 'realidad suprasensible' y que por eso no debe confundirse con el uso del término en el sentido de la metafísica clásica, que se refiere a la realidad espacio-temporal de los objetos naturales.

\footnotetext{
12 “Por 'realismo lógico' entiendo (...) la doctrina según la cual la lógica (incluida la teoría del conocimiento) tiene que ver con una categoría propia de objetos, que son realmente (real) diferentes tanto de los fenómenos psíquicos como de las expresiones lingüísticas" (Morscher 1972, p. 69).

13 Alberto Coffa sostiene la idea de que en la tradición epistemológica del siglo XIX se destacaron tres corrientes, cuyo eje de divergencia se definió a partir de su relación con el concepto de 'a priori': el positivismo (que lo negaba), los (neo)kantianos (que lo remitían a la subjetividad trascendental), y la tradición semántica que "creía en el a priori pero no en los poderes constitutivos de la mente" (Coffa 1991, p. 1). Para estos últimos la "semántica, y no la metafísica, era su prima philosophia" (Coffa 1991, p. 2).

14 Cf. Smith 1996, p. 156.

15 Michael Dummett distingue dos tipos de realismo: uno se opone al idealismo y otro se opone al nominalismo; al primero podríamos llamarlo 'realismo metafísico' y al segundo 'realismo platónico' (las denominaciones no son de Dummett, pero sí su caracterización). El elemento común consiste en la postulación de la independencia ontológica de ciertas entidades, sean ya de los objetos materiales (realismo metafísico) o de los universales (realismo platónico) (cf. "Realism", pp. 145-146; cf. "Platonism", en Dummett 1978).
} 


\section{La diferencia entre lógica y psicología en la obra de J. F. Herbart}

Johann Friedrich Herbart (1776-1841) es en algún sentido más recordado como uno de los padres de pedagogía en tanto disciplina independiente, que como filósofo. 16 Una de sus publicaciones filosóficas más conocidas es el Lehrbuch zur Einleitung in die Philosophie (Manual de introducción a la filosofía). Publicada por primera vez en 1813 , es una obra que en su estructura podría considerarse clásica17 y que fue concebida por su autor como un texto introductorio para los estudiantes, cuya función era dar los rudimentos fundamentales de la filosofía y además llevarlos a pensar por sí mismos. En cualquier caso, su figura filosófica fue opacada en buena medida por el impacto del idealismo alemán, de gran vigencia en el mundo académico teutón en la época de actividad de Herbart. Hegel y, en general, los idealistas alemanes fueron sus principales rivales. ${ }^{18} \mathrm{Su}$ manual es una obra extensa (consta de 164 parágrafos) que puede analizarse en niveles muy diferentes. Siguiendo la finalidad del presente artículo, sólo nos interesa remarcar dos aspectos de su filosofía: la separación categórica entre la lógica y la psicología, y la base platónica que sustenta dicha distinción.

En el prefacio a la Cuarta Edición del Lehrbuch, Herbart señala el agregado de un nuevo capítulo -en esta nueva edición- sobre la aplicación de la lógica (la lógica aplicada), cuya meta es evitar la "precipitada intromisión de la psicología en la lógica" (Herbart 1837, p. 27). Herbart profundiza y aclara esta idea en la Segunda Sección titulada "La Lógica", al sostener que una cosa es reconocer la existencia de aspectos psicológicos propios de nuestra naturaleza mental, es decir, de actos de pensamiento que nos permiten descubrir ciertas verdades, y otra muy distinta es confundir dichos actos de pensamiento con aquellos pensamientos en sí mismos - esta idea será luego desarrollada por Bolzano con mucha más precisión-. Así, afirma Herbart que: "Todos nuestros pensamientos se pueden considerar desde dos perspectivas diferentes; en parte en tanto actividades de nuestro espíritu (Geist), en parte con relación a aquello que se puede pensar a través de ellas [i.e. a través de las actividades mentales]. Estos últimos se llaman conceptos (Begriffe), palabra que -en la medida en que denomina lo concebido (Begriffene ${ }^{19}$ ) - exige realizar una abs-

\footnotetext{
16 Obras como las Lecciones de Pedagogía (editadas en 1835 y luego en 1841) pueden considerarse como uno de los textos fundacionales de la ciencia pedagógica (cf. Herbart, J. F., Umriss pädagogischer Vorlesungen, Paderborn, Schöningh, 1957).

17 La misma consta de cuatro partes: la Propedéutica, la Lógica, la Estética o Filosofía de los valores y la Metafísica.

18 En el Prefacio a la segunda edición, Herbart muestra una preocupante sorpresa por aquéllos que erróneamente le atribuyen a su filosofía expresiones propias de las filosofías de Spinoza, Fichte o Schelling, con quienes, "como es sabido", no tiene ninguna afinidad (Herbart 1837, p. 22).

19 En alemán 'Begriffene' es la sustantivación del participio del verbo 'begreifen' (comprender, concebir, captar, etc.), a partir del cual se deriva la noción de 'Begriff' (concepto). Por lo tanto, 'das Begriffene' puede traducirse por 'lo captado' o 'lo concebido', y aquí debemos hacer especial hinca-
} 
tracción del modo en que recibimos, producimos o reproducimos los pensamientos (Gedanken)" (Herbart 1837, pp. 81-82). Así, sostiene Herbart: "En la lógica es necesario ignorar todo aquello que sea psicológico, puesto que en este marco sólo deben determinarse aquellas formas de la conexión posible de lo pensado (Gedachten), que admite lo pensado mismo según su propiedad (Beschaffenheit)" (Herbart 1837, p. 82). Incluso, en una nota al pie del mismo parágrafo citado, Herbart señala que no sólo es indispensable la separación de la lógica y de lo psicológico, sino que asimismo hay que separar la lógica (pura) de la lógica aplicada (cf. Herbart 1837, p. 81, nota 1).

Esta profunda y categórica separación entre la lógica (lo pensado) y la psicología (el pensar o el pensamiento) conlleva una consecuencia muy importante, que marca la adscripción a un cierto tipo de realismo platónico por parte de Herbart: "La primera consecuencia de esta explicación [es decir, de la cita anterior] es el principio según el cual dos conceptos no pueden ser completamente iguales, sino que cada uno, de algún modo, sólo se encuentra (vorhanden ist) en un único ejemplar. Pues dos conceptos iguales no se diferenciarían con relación a aquello que es pensado a través de ellos; en tanto conceptos pues no se diferenciarían en absoluto. Por el contrario, el pensamiento de un mismo concepto puede repetirse muchas veces, producirse y evocarse en circunstancias muy diferentes, y retomarse por innumerables seres racionales, sin que el concepto, en este sentido, se multiplicase" (Herbart 1837, p. 82; la cursiva es mía). En otras palabras: aquello que se piensa es una unidad inteligible que se diferencia claramente de la multiplicidad de los actos de pensamiento humano.

Cuando se forja un pensamiento y se pasa del mero concepto (cf. Herbart 1837, Segunda Sección, Capítulo 1) al juicio (Segunda Sección, Capítulo 2), nos encontramos con la misma situación que en los casos anteriormente indicados: el pensamiento que se expresa a través de los juicios no es otra cosa que la exteriorización de la unión de determinados conceptos. En otras palabras: unir predicativamente dos o más conceptos en un juicio no reduce en absoluto el contenido conceptual a un mero pensamiento judicativo. El valor lógico de la proposición no puede identificarse con el hecho psicológico de un juicio. Como veremos, dichas ideas se tornan más explícitas en la obra de Lotze (y por supuesto en la de Frege). Así, afirma Herbart: "Pero el pensamiento es aquí sólo el medio, por así decir, el vehículo para juntar los conceptos; depende de ellos mismos [i.e. de los conceptos] si son compatibles o no. Por ello, aquí también se debe distanciar claramente lo lógico de toda intromisión de lo psicológico" (Herbart 1837, p. 96).

La misma idea se hace extensiva a la psicología genética: así como la lógica debe separarse de la psicología, "las investigaciones metafísicas no deben mezclar-

pié en el uso del artículo neutro, puesto que dicha palabra no hace referencia al acto de concebir / captar, sino a aquello que es concebido / captado. 
se con una mirada retrospectiva al origen de nuestras representaciones" (Herbart 1837, p. 228). En otras palabras la metafísica, en tanto descripción de los conceptos fundamentales (Grundbegriffe), no debe confundirse con la investigación en torno al origen psico-genético de nuestras representaciones; es decir, toda investigación genético-psicológica que pretenda explicar y justificar la validez lógica de los conceptos fundamentales a partir de su origen empírico genético, debe quedar fuera del ámbito de fundación filosófico-metafísica fundamental. Aquí podemos observar un claro gesto de sesgo platónico: la ciencia fundamental se basa en conceptos que son unidades ideales de sentido que no son empíricas (ni en sentido externo, ni en sentido interno). Siguiendo esta línea, ya desde el $\S 1$ la filosofía es definida en tanto trabajo con conceptos (cf. Herbart 1837, § 1).

Ahora, ¿en qué medida y en qué sentido podemos entender y definir su platonismo? Por un lado, debemos señalar la consideración histórica que Herbart hace del mismo. Por otro lado, es fundamental evaluar su platonismo a nivel conceptual -que es lo que aquí más nos interesa-. A nivel histórico la relación es explícita: así los filósofos eleáticos y Platón sostenían que "sólo un ser inmutable puede constituir (ausmachen) el objeto del saber" (Herbart 1837, p. 230). La altísima valoración de la figura de Platón en el marco de la historia de la filosofía se hace patente ya en el Prefacio de la Segunda Edición, cuando Herbart explica cuál debería ser el recorrido de la historia de la filosofía para un principiante y cuáles deberían ser las grandes figuras que éste debería seguir: en el marco de la filosofía antigua es sin dudas Platón el filósofo a estudiar y en segundo lugar Sexto Empírico (cf. Herbart 1837, p. 25). Su reconocimiento a Platón se torna más explícito en la Cuarta Sección, donde le dedica todo el Capítulo 4 a las ideas platónicas, definidas en tanto cualidades absolutas (absolute Qualitäten) (cf. §§ 143-148). Para Herbart, la recepción histórica de la filosofía de Platón es fruto de un error (cf. Herbart 1837, p. 250, 252). Es precisamente aquí donde, más allá de su análisis histórico del platonismo, debemos dirigir nuestra atención, concentrándonos en las consideraciones de tipo conceptuales que conforman la base del pensamiento metafísico herbartiano. La intención de Herbart es así definir el significado auténtico de las cualidades (Qualitäten) o esencias, que éste identifica con las ideas platónicas.

En este contexto, Herbart define su relación con el 'platonismo histórico': la genialidad de Platón consistió en su convicción de que el objeto del auténtico saber consiste en las ideas; de allí en adelante el principal error histórico -señalado en el Capítulo 4 de la Cuarta Sección del Lehrbuch- fue la mala interpretación por parte de la tradición del verdadero significado del platonismo, en el preciso momento en que "la palabra [idea] (...) recibió el significado de representaciones (Vorstellungen) de cualquier ser pensante" (Herbart 1837, p. 252; cf. p. 253). En otras palabras, el error de la tradición fue confundir la realidad (inteligible) objetiva de las ideas con su representación (o copia) mental en sentido psicológico (i.e., 
idea en tanto imagen o contenido mental). El objeto del conocimiento no son las representaciones (Vorstellungen) mentales, sino las ideas en el sentido absoluto y realista (platónico) del término. Nos encontramos así con una diferencia fundamental entre 'lo inteligible' y 'lo sensible'. Al igual que Platón, Herbart reconoce las múltiples complicaciones que conlleva la relación (o participación) entre las ideas y las cosas, tema que aquí no nos compete. Lo importante es señalar que las ideas (inteligibles) son definidas en tanto objetos reales (reale Gegenstände), que son auténticos modelos (Vorbilder, Muster, paradeígmata); es decir, una idea es una cualidad absoluta (eidos) (cf. Herbart 1837, pp. 254-255).

Una cuestión fundamental que debemos mencionar es la consideración que efectúa Herbart con relación al concepto de 'realidad' utilizado en relación a las ideas platónicas. Dicho concepto no debe entenderse en el sentido de 'realidad natural': "Para entender los escritos de Platón es menester señalar que aquí -donde no hay que pensar en absoluto en una auténtica doctrina natural (puesto que todo lo que implique cambio ha quedado excluido)- las consideraciones lógicas (...) deben ocupar absolutamente el lugar de las consideraciones físicas" (Herbart 1837, p. 257). En tal sentido, sostiene Herbart -quizás de un modo un tanto tosco, i.e. sin la precisión conceptual que luego tanto Bolzano como Lotze le darán a este términoque hay aceptar la realidad de las ideas y la validez de los conceptos modélicos (Gültigkeit der Musterbegriffe) (cf. Herbart 1837, p. 257).

En pos de una justa evaluación, hay que reconocer, en primer lugar, que el Lehrbuch es sólo una introducción a la filosofía y, en segundo lugar, que Herbart estaba más interesado en la función pedagógica del manual que en posibles discusiones filosóficas minuciosas. No obstante, debemos reconocer que en el curso de introducción a la filosofía de Herbart -que fue un manual de estudio muy extendido en el mundo germano- se puede ya entrever la presencia de los dos elementos esenciales que son el tema del presente artículo: el platonismo, interpretado a partir del realismo absoluto de las ideas, y el antipsicologismo que, a partir de la base ontológica que le brinda dicho platonismo, puede entenderse como la idea de que lo lógico tiene que ver con entidades absolutas y no con contenidos o representaciones mentales.

\section{Bernard Bolzano y el 'en sí'}

Bernard Placidus Johann Nepomuk Bolzano (1781-1848) fue uno de los más grandes filósofos austriacos (de Bohemia, actualmente perteneciente a la República Checa). De extraordinaria formación académica, no sólo fue sacerdote católico, sino además filósofo, matemático, lógico y teólogo. Fue un auténtico 'libre pensador' que opinó sobre las áreas más audaces y controvertidas, algo que lo llevó a ser 
acusado de herejía en 1819 y a ser sometido a un proceso. Su profundidad filosófica y el volumen de su ingente obra -cuya edición crítica consta de más de 120 volúmenes- no se condicen con el reconocimiento histórico merecido, formando parte, como tantos otros, de las figuras más injustamente olvidadas de la filosofía del siglo XIX. Su gran obra fue la ingente Wissenschaftslehre (Doctrina de la ciencia) publicada en 1837.

En cualquier caso, directa o indirectamente, no es difícil rastrear la influencia que dejó su obra hasta los orígenes mismos de la fenomenología y de la filosofía analítica. La Philosophische Propädeutik de Robert Zimmermann, discípulo de Bolzano, fue una obra publicada en Viena en 1853 que resumía muchas de las ideas de la Wissenschaftslehre; dicha Propedéutica fue el principal manual de filosofía en el Imperio Austro-Húngaro. De aquí puede trazarse una influencia de Bolzano tanto en Brentano -quien por otra parte rechazaba el platonismo de Bolzano-, como en muchos de sus discípulos (Twardowski, Höfler, Meinong, Kerry, entre otros), pero particularmente en la obra de Edmund Husserl, quien reconoce explícitamente la influencia bolzaniana, como queda muy claro por lo menos en la etapa de nacimiento de la fenomenología. ${ }^{20}$ También pueden trazarse ciertas influencias directas e indirectas en la filosofía analítica ${ }^{21}$ y en la tradición lógica polaca-Twardowski, Leśniewski y Tarski22-. Es difícil definir a nivel histórico-filológico si hubo una eventual influencia en Frege. Si bien hay numerosos indicios a nivel conceptual, no hay -al menos en lo que respecta a referencias directas- elementos concluyentes. ${ }^{23}$ Es interesante resaltar en este contexto la lectura de Barry Smith, quien sostiene que, si bien el objetivismo lógico (platónico) tuvo una marcada influencia de Bolzano (quizás vía Zimmermann) en el mundo austriaco, paralelamente, en Alemania se desarrolló una corriente de objetivismo lógico similar, pero a partir de la obra de Lotze -y quizás podríamos agregar a Herbart, quien hubo antecedido a Lotze en la cátedra de Göttingen-, y que ésta sí, muy probablemente, tuvo una influencia en la obra de Frege24; por supuesto, deberíamos incluir aquí también al mismo Husserl, quien reconoce esto explícitamente.

La Wissenschaftslehre constituye una auténtica enciclopedia filosófica conformada por un ingente universo conceptual con una casi perfecta organización arquitectónica. Aquí simplemente nos concentraremos en aquellos aspectos que son relevantes para nuestro tema principal, en particular, su análisis de los objetos lógicos.

\footnotetext{
20 Esto es patente en el caso de las Lecciones dictadas en Halle en 1896 (cf. Husserl, E., Logik. Vorlesung 1896, Materialien I, Dordrecht, Kluwer, 2001), así como en las Logische Untersuchungen. ${ }^{21}$ Michael Dummett cuenta que Gilbert Ryle fue uno de los primeros en introducir la obra de Bolzano junto con las de Brentano, Husserl, Frege, y otros 'filósofos continentales' en el mundo anglosajón (cf. Dummett 1993, p. ix).

22 Cf. Simons 1992, p. 14.

23 Cf. supra, nota 2.

${ }^{24}$ Cf. Smith 1996, p. 156, nota 3.
} 
Dichos 'objetos' se inscriben en el marco de lo que podríamos denominar 'realismo semántico', en tanto Bolzano entiende que estas 'entidades' no dependen en absoluto de ningún tipo de determinación óntico-ontológica, sino que 'son' o, mejor dicho 'valen' (en el sentido de 'es gilt', de la 'Geltung') con absoluta independencia del ser de los entes (cósicos). Estas 'entidades' -que podríamos denominar 'semánticas' en la medida que son unidades de sentido- son: las 'representaciones en sí' (Vorstellungen an sich) y las 'proposiciones en sí' (Sätze an sich) -entre las que hay que incluir como una suerte de sub-categoría de las mismas a las 'verdades en sí' (Wahrheiten an sich)-. Podríamos decir que las representaciones en sí son auténticas ideas en el sentido platónico del término, mientras que las proposiciones en sí son las combinaciones sintácticas de las mismas, siendo algunas verdaderas y otras falsas ( $\sin$ por eso dejar de ser 'en sí'). Dichas 'entidades' no son objeto de la psicología (pues no se trata de representaciones mentales), ni a fortiori de ninguna ciencia material. Pero lo complejo del análisis bolzaniano consiste en que tampoco dependen ni de la ontología ni, llamativamente, de la lógica, porque stricto sensu no se trata de objetos. El caso más emblemático es quizás el de las 'representaciones sin objeto' (Gegenstandslose Vorstellungen), como, por ejemplo, las contradicciones: éstas no tienen objeto y ni siquiera pueden incluirse en el terreno de la lógica, sin por ello, no obstante, dejar de tener un sentido ideal; tampoco son entidades lingüísticas, en tanto no dependen de un determinado lenguaje.

En pocas palabras: los componentes fundamentales de la Wissenschaftslehre son estas 'entidades en sí'. Lo particular de esta interpretación y que le da su tinte platónico es precisamente la idea del 'en sí' (an sich), es decir, la absoluta independencia de cualquier otra entidad (sea mental, ontológica, etc.) que no sea sí misma.

Sin lugar a dudas, puede decirse que de este modo Bolzano anticipa claramente la refutación al psicologismo. ${ }^{25} \mathrm{Si}$ bien Bolzano, al igual que Herbart, reconoce cierto valor a la psicología y al hecho que las verdades que se presentan (lo pensado en sí) están de hecho relacionadas con el pensamiento (i.e. los procesos mentales por los que conocemos las verdades), no obstante, una de las tesis centrales de Bolzano consiste en separar tajantemente las reglas (subjetivas) del pensamiento (Denken) de lo pensado (Gedachte) en sí, es decir, de los contenidos (Inhalte) (objetivos) que se piensan (cf. Bolzano 1837, p. 25). La verdad en sí (así como en general toda proposición en sí e incluso todo representación en sí) constituye una dimensión ideal de sentido, por lo que ésta de ningún modo se puede identificar con los actos de pensamiento a través de los cuales llegamos a pensar lo pensado.

El $\S 12$ de la Wissenschaftslehre define claramente su antipsicologismo, en la medida que plantea la posibilidad de una lógica cuyo objeto no sean las 'verdades pensadas', sino las 'verdades generales' (Wahrheiten überhaupt). Y yendo incluso más allá del problema de la verdad o falsedad de una proposición, plantea la posi-

25 Cf. George 2003 
bilidad de una ciencia cuyo objeto no sean las 'proposiciones pensadas', sino las 'proposiciones en sí' simpliciter, es decir, no sólo con total independencia de que sean pensadas o no, sino además con independencia de que sean verdaderas o no (cf. Bolzano 1837, p. 77). El hincapié cae sólo en la validez (Gültigkeit) de las mismas. El antipsicologismo de Bolzano se resume en las siguientes palabras: "mostraremos que la fuente de la mayoría de los errores que se han presentado hasta ahora en la lógica, residen en el hecho de que (...) no se distinguió con suficiente claridad [en la tradición] entre las verdades pensadas (gedachte Wahrheiten) y las verdades en sí (Wahrheiten an sich), [y por otra parte entre] las proposiciones y los conceptos pensados (gedachte Sätze und Begriffe) y las proposiciones y los conceptos en general (Sätze und Begriffe überhaupt)" (Bolzano 1837, p. 47). Bolzano atribuye incluso muchos de los errores de la lógica tradicional a la lamentable confusión entre los objetos ideales y sus modos de aparecer (que remiten a modos de pensar) (cf. Bolzano 1837, p. 61). Los objetos ideales "valen (gelten) para todos los seres (incluso para Dios mismo)" (Bolzano 1837, p. 64).

Bolzano no sólo reconoce explícitamente la dificultad de dar una definición directa de estas 'entidades', sino que además cree que tampoco es siempre aconsejable intentar definirlas (cf. Bolzano 1837, p. 91). En cualquier caso, se puede hacer un intento mereológico de explicación (en el sentido de la teoría de 'todos' y 'partes'): las proposiciones en sí (Sätze an sich) son 'todos' formados por las representaciones en sí (Vorstellungen an sich), que son 'partes' que componen aquellos 'todos'; en otras palabras, la representación es una 'parte' de un 'todo' que es la proposición. ${ }^{26} \mathrm{~A}$ su vez, dichas proposiciones en sí, pueden ser tanto 'proposiciones verdaderas' (wahre Sätze) como 'proposiciones falsas' (también consideradas en su 'en sí', sin por ello hacer mella en su valor semántico ideal) (cf. Bolzano 1837, §§ 19-23). Es decir, las 'verdades en sí' son proposiciones en sí que (además) son verdaderas. Esto implica ipso facto que puede haber proposiciones en sí falsas (por ejemplo, 'el cuadrado es redondo'), que no por ello dejan de tener sentido.

Mientras que las proposiciones en sí tienen una estructura predicativa, por ejemplo: 'Dios es todopoderoso', las representaciones en sí carecen de dicho carácter predicativo y sólo representan un contenido conceptual, por ejemplo: 'Dios todopoderoso'. Ambas son, por supuesto, 'entidades' ideales que valen por sí mismas independientemente de que exista o no un correlato 'existente' (en este caso, Dios) que tenga el atributo de ser todopoderoso. Es menester además señalar, como enfatiza constantemente Bolzano, que la representación en sí no debe entenderse en tanto contenido o imagen mental (cf. Bolzano 1837, pp. 215-217), como lo hizo erróneamente buena parte de la tradición filosófica; en este punto, coincide claramente con Herbart.

26 “" $[\mathrm{P}]$ or representación en significado objetivo (Vorstellung in objektiver Bedeutung) o representación en sí entendemos algo a partir de lo cual (...) se compone una proposición en sí, así como un todo [se compone] de sus partes" (Bolzano 1837, p. 99). 
En el marco de este complejo análisis conceptual de Bolzano, lo más importante para nosotros es rescatar la idea de la 'no-existencia' de dichas 'entidades'. El 'en sí' (an sich) del que habla Bolzano no existe, es decir, carece de existencia real; por eso, Bolzano apela al uso del verbo 'hay' (es gibt): entonces decimos, por ejemplo, 'hay verdades en sí' (es gibt Wahrheiten an sich). De este modo, excluimos eo ipso toda cuestión concerniente a la existencia óntica de dichas 'entidades' (cf. Bolzano $1837, \S 30)$. En otras palabras, estas unidades ideales de sentido 'en sí' son independientes de la ontología y de la lógica, y constituyen un orden de 'realidad' similar al que Platón atribuía a su topos ouranós.

\section{El platonismo de R. Hermann Lotze}

Así como Bolzano, Rudolf Hermann Lotze (1817-1881) comparte la misma suerte de otros grandes pensadores del siglo XIX de haber sido casi totalmente olvidado por la tradición filosófica del siglo XX. Como señala correctamente Orth y como puede observarse en las numerosas referencias a su obra en los escritos de muchos filósofos de fines del siglo XIX y principios del XX, Lotze fue uno de los filósofos más citados, comentados y discutidos entre 1875 y finales de la Primera Guerra Mundial. ${ }^{27}$ Fue una de las figuras principales y más reverenciadas de la universidad alemana de la segunda mitad del siglo XIX; y dicho reconocimiento no se circunscribe al mundo germánico: su repercusión fue muy importante también en el mundo anglosajón. ${ }^{28} \mathrm{Su}$ influencia - directa o indirecta- puede observarse en pensadores muy heterogéneos entre sí como W. Dilthey, F. Brentano, O. Liebmann, W. James, C. Stumpf, G. Frege29, E. Husserl, M. Scheler, E. Lask, etc. ${ }^{30}$

$\mathrm{Su}$ formación intelectual es heterogénea: además de haberse graduado en filosofía y en medicina (llegó incluso a ejercer como médico), era muy versado en biología, psicología y lógica. Si bien su obra tiene un innegable carácter especulativo, no por ello deja de atender a la experiencia concreta en sus más diversas manifestaciones; de este modo, su filosofía se extiende a una gran amplitud de temas. En algún sentido, Lotze fue un hijo fiel del pensamiento clásico alemán, en tanto su intención era construir un auténtico sistema filosófico que explique las más variadas áreas de la realidad. En dicho marco se inscribe su primer gran proyecto (inconcluso) de un Sistema de filosofia que consta de tres partes. La primera parte era la Lógica (Primera Edición: 1843, corregida y reeditada luego) que constaba de tres

\footnotetext{
27 Cf. Orth 1986, p. 7.

28 Cf. la Introducción de D.W. Simon, en Stählin 1889, p. vii y ss.

29 En torno a una eventual influencia de Lotze en la obra de Frege se desató una aireada polémica entre Michael Dummett y Hans Sluga, como ya se señaló más arriba en la nota 2.

30 Cf. Orth 1986, p. 8; cf. Gabriel 1989a, p. xii.
} 
libros: 'Del pensamiento', 'Del investigar' y 'Del conocer' (aquí haremos hincapié en este tercer libro). La segunda era la Metafisica (Primera Edición: 1841, también luego corregida y reeditada) que, siguiendo la tradición clásica de Wolff, se dividía en 'Ontología', 'Cosmología' y 'Psicología'. La tercera parte del sistema era la Ética, cuya división era 'Filosofía práctica', 'Estética' y 'Filosofía de la religión'; esta tercera y última parte no llegó a ser publicada. Cabe además mencionar entre sus grandes obras el Microcosmos: Ideas sobre la historia de la naturaleza y la historia de la humanidad, publicada entre los años 1856 y 1864.

Como ya anticipamos, aquí sólo nos interesa el tercer libro de la Lógica, es decir, 'Del conocer' (Vom Erkennen). Allí Lotze plantea un claro rechazo al psicologismo, que se sustenta en su original interpretación de la teoría de las ideas de Platón, desarrollada en el capítulo denominado: "El mundo de las ideas" (Die Ideenwelt). ${ }^{31} \mathrm{Al}$ igual que Herbart, Lotze toma claramente posición del lado antipsicologista; un núcleo central de esta posición es la distinción entre las 'pretensiones de explicación' (Erklärungsansprüche) de la psicología y las 'pretensiones de validez' (Geltungsansprüche) de la lógica. Gottfried Gabriel lo define así como uno de los padres de la crítica al psicologismo. 32

La Introducción al primer libro de la Lógica se titula 'Del pensar' (Vom Denken). Lotze señala desde un comienzo que su análisis no tiene como objeto el pensar en tanto actividad cognitiva, sino que su interés se dirige a las formas y leyes puras y reales del pensamiento (cf. Lotze 1874, p. 9 y ss.). 33 Siguiendo una distinción que remite a Kant ${ }^{34}$, Lotze distingue claramente el 'contenido' (Inhalt) de la 'historia de su génesis' (Entstehungsgeschichte); en otras palabras, una cosa es la validez lógica de algo y otra cosa muy distinta es su origen, su génesis. El 'conte-

\footnotetext{
${ }^{31}$ La obra de Herbart tuvo una gran influencia en la filosofía de Lotze (cf. Orth 1986, p. 13; cf. Gabriel 1989b, p. xi, nota 9). En este contexto, es quizás relevante señalar que Lotze ocupó el lugar que Herbart había dejado como profesor en la Universidad de Göttingen, en la cual Frege estudió y Husserl enseñó después. Es interesante mencionar como dato histórico, que durante el período en el que Husserl fue docente en Göttingen (de 1901 a 1916) varios de los llamados 'fenomenólogos de Munich' abandonaron esta ciudad y emigraron para estudiar con Husserl en la Universidad de Göttingen; en dicho lugar, se constituyó así un importante centro de fenomenología realista antipsicologista (tal es el caso por ejemplo de Adolf Reinach). Este dato es relevante, en tanto señala cierta línea de continuidad intelectual de dicha universidad.

32 Cf. Gabriel 1989b, p. ix. Gabriel sostiene además que fue especialmente su Logica la que tuvo un gran impacto en el mundo académico alemán, en particular su "decidida interpretación de la validez lógica de la doctrina platónica de las ideas" (cf. Gabriel 1989b, p. x), mientras que en el mundo académico británico fue su Metafisica la que tuvo mayor repercusión, principalmente en el marco del hegelianismo inglés.

33 Las citas de Lotze se harán siguiendo la edición de 1874 de la Lógica, corregida en mínimos detalles en 1928, y que conserva su paginación original. Ésta es la edición seguida en los tomos re-editados por G. Gabriel (cf. Gabriel 1989a, p. viii y Gabriel 1989b, p. vii).

34 Cf. Vigo 2013, pp. 20 y 22.
} 
nido' no es otra cosa que la "lógica pura o formal" del "pensamiento general" y las "formas y principios fundamentales generales" (Lotze 1874, p. 12). Cabe aclarar que Lotze no desvaloriza el estudio (psicológico-genético) de la formación de conceptos - de hecho, algunas de sus investigaciones analizan la formación genética de conceptos que van desde la sensibilidad hasta las formas lógicas-; pero en el marco de una auténtica 'lógica de la validez' el eje debe centrarse en las unidades lógicas de sentido, en tanto entidades independientes de lo real-sensible, de lo mental, etc. El uso del lenguaje para nombrar dichas estructuras lógicas no les quita a éstas su 'consistencia' (Bestehen) propia ni su independencia de todo ser pensante (cf. Lotze 1874, p. 16).

En el tercer libro de la Lógica, sobre el conocer (Erkennen), se analizan problemas relacionados con la lógica y la teoría del conocimiento, tales como el escepticismo, el apriorismo, las verdades a priori, etc. En este contexto, la doctrina de las ideas (en sentido platónico) ocupa un lugar central. Es decir, hay una anterioridad de principio de la lógica de la validez con relación a las explicaciones psicológicogenéticas. Estas últimas sólo pueden explicar el origen empírico del conocimiento, pero no legitimar su validez. ${ }^{35}$ Así, colocar la psicología en la base del conocimiento conduce casi inexorablemente a una forma de escepticismo. 36

Lotze dedica un capítulo completo al problema del escepticismo. La refutación consiste en explicitar primero los presupuestos inexorables de todo escepticismo, para luego mostrar que dichos presupuestos lo llevan a caer inevitablemente en inconsecuencia consigo mismo. Se puede bosquejar brevemente su argumento del siguiente modo (cf. Lotze 1874, p. 485 y ss.): nadie duda que existe el error y que éste atraviesa el conocimiento humano; en tal sentido, está claro que el descubrimiento de nuevas verdades siempre es posible y el conocimiento es siempre perfectible; por ende, el descubrimiento de verdades es una "empresa interminable" (Lotze 1874, p. 496). Ahora, una negación absoluta de la posibilidad del conocimiento (en el sentido del malie génie cartesiano) no es posible, puesto que la duda misma sólo es posible bajo el presupuesto de alguna verdad reconocida. Esto nos lleva a plantear las siguientes preguntas: ¿cómo podríamos saber qué estamos negando si no tenemos al menos una manifestación de algo que creemos y consideramos verdadero? ¿Cómo sostener una duda radical de algo, de lo cual ni siquiera sabemos qué es? En la misma consideración de algo que se pone en duda se presupone al menos la idea de dicho 'algo', así sea como mero punto de partida. Esto nos

35 "En ningún lugar de mi exposición sostuve la posición de que la lógica pueda sacar algún provecho esencial de la investigación sobre las condiciones en las cuales el pensamiento se materializa en tanto proceso psíquico. El significado de las formas lógicas consiste en el sentido de las conexiones" (Lotze 1874 , p. 543).

36 Lotze además señala que todo intento de fundar la lógica en la psicología (psicologismo) cae indefectiblemente en un círculo, en tanto el fundamento que se da a la lógica desde la psicología presupone las mismas leyes (lógicas) que se pretenden justificar (cf. Lotze 1989b, p. 543). 
conduce a una forma de racionalidad básica de la vida cotidiana; así, en lo más elemental, siempre hay una confianza en nuestra razón y su relación con el mundo. Lotze denomina a esto "autoconfianza de la razón" (Selbstvertrauen der Vernunft) (Lotze 1874, p. 489) y concluye: "la autoconfianza de la razón, que la verdad en general puede ser encontrada a través del pensar, es el presupuesto inevitable de todo investigar" (Lotze 1874, p. 492). De hecho, uno puede preguntarse siguiendo a Lotze, ¿cómo podemos siquiera iniciar una investigación (en filosofía o en cualquier otra área del saber humano), si no creemos que lo que estamos diciendo, sosteniendo y defendiendo (por más escéptico y relativista que sea el argumento) es verdadero? (Cf. Lotze 1874, p. 498-499).

En todo caso, hay verdad, es decir, proposiciones verdaderas. Pero el valor adicional de la filosofía de Lotze consiste no sólo en el reconocimiento de dicha verdad y en su concomitante refutación del escepticismo, sino en el valor en sí de dicha verdad; es decir, ésta no depende ni de las representaciones mentales subjetivas, ni de una presunta existencia real en sentido óntico de aquello a lo que la verdad refiere. Como podemos observar, el eje de la cuestión cae sobre el estatuto ontológico de este 'ente' portador de la verdad -en términos contemporáneos podríamos hablar de 'portador de verdad' (truth-bearer)-. Es precisamente aquí cuando Lotze introduce su original interpretación de la teoría de las ideas de Platón. La base ontológica platónica que presenta es mucho más compleja que la de Herbart y más explícita que la de Bolzano. ${ }^{37}$ Lotze desarrolla así su teoría de la 'lógica de la validez' (Geltungslogik), que se funda en su teoría de las ideas de Platón, como veremos a continuación.

El capítulo sobre la teoría de las ideas comienza con una referencia a Heráclito $\mathrm{y}$ sus ideas de que todo fluye (panta rei) y de que nunca entramos dos veces al mismo río. A partir de esto, Lotze busca radicalizar la hipótesis del perpetuo cambio: la constatación de que en nuestra vida cotidiana todo a nuestro alrededor cambia, es casi una verdad de Perogrullo que ni siquiera necesita ser probada; ahora bien, si todo, pero absolutamente todo, cambia, incluso nuestras verdades más sólidas también deberían cambiar, con lo que nos encontraríamos ante un verdadero inconveniente teórico. El razonamiento pasa a ser entonces consecuencialista: si absolutamente todo cambia, entonces sólo nos queda pensar en una inconsistencia (Unbeständigkeit) general de toda determinación de pensamiento; pero esta conclusión implicaría ipso facto que "todo contenido conceptual significa a la vez lo que quiere decir y a la vez lo que no quiere decir" (Lotze 1874, p. 506), o sea, se caería indefectiblemente en una serie de contradicciones inconsecuentes. A partir de este

\footnotetext{
${ }^{37}$ Husserl sostiene que su teoría de la idealidad de las significaciones, de las representaciones y de los contenidos de juicio, más allá de la clara influencia de Bolzano, viene de Lotze y de su genial interpretación de la teoría de las ideas de Platón (cf. Husserl 1979, p. 156).
} 
marco conceptual de incertidumbre absoluta, según Lotze, Platón llega a su teoría de las ideas, cuya verdad es absolutamente independiente del ser de las cosas.

Aquí yace el punto de partida de la filosofía de Lotze en su interpretación de Platón: no todo lo que es, es en el sentido del ser o existir de las cosas; en otros términos: no toda la realidad se reduce al ser en sentido óntico o existencial. El concepto de 'realidad' (Wirklichkeit) es para Lotze un género ontológico supremo. E1 concepto de 'ser' (Sein), en el sentido de 'existir' (Dasein), es sólo un modo de realidad. Esto quiere decir que el concepto de 'realidad' es más amplio que el de 'ser', abarcando otros modos de realidad que no necesariamente son modos de ser -aquí podemos observar ciertas analogías con Bolzano-. En tal sentido, explicando el alcance de dicho concepto de 'realidad' (Wirklichkeit), sostiene: 'Llamamos real (wirklich) a una cosa (Ding) que es (ist), en contraposición a otra que no es; también llamamos real a un acontecimiento (Ereignis) que sucede (geschieht) o que ha sucedido, en contraposición con uno que no sucede; [1lamamos también] real a una relación (Verhältnis) que tiene consistencia (besteht), en contraposición con aquella que no la tiene; por último llamamos verdaderamente real a una proposición (Satz) que tiene validez (gilt), en contraposición con aquella cuya validez (Geltung) es dudosa" (Lotze 1874, p. 511, la cursiva es mía). Podemos observar aquí al menos cuatro 'realidades' diferentes: 1) el ser (Sein) en tanto ser cósico; 2) el suceder en tanto acontecimiento (Ereignis, Geschehen); 3) el consistir o -para evitar un giro extraño al idioma castellano- el tener consistencia (Bestehen); y 4) el valer o tener validez (Geltung).

Dicha clasificación de 'lo real' impone condiciones categoriales: así, predicar un tipo de realidad a otro tipo que no es el que le corresponde, lleva directamente a lo que podría denominarse 'error categorial', es decir, a confundir categorías no homologables. En tal sentido, no podemos afirmar con consistencia lógicocategorial que una 'proposición (Satz) es (ist)', puesto que sólo las cosas (Dinge) son. Una proposición sólo vale (gilt), es decir, su realidad reside precisamente en su valor de verdad. 38 Por dicho motivo, no tiene ningún sentido preguntarse por el 'ser' (Sein) -en sentido cósico- de una proposición, puesto que de la misma no se puede predicar ni que es, ni que no es, en tanto dichos predicados no corresponden a tal categoría. Muchos errores categoriales a lo largo de la historia de la filosofía podrían haberse evitado de haberse atendido a dichas distinciones conceptuales (cf. Lotze 1874, p. 512).

En lo que respecta a nuestro interés principal, debemos concentrarnos particularmente en el concepto de 'validez' (Geltung). A partir de lo afirmado, debe que-

\footnotetext{
38 Aquí podemos observar ciertas analogías con Frege, para quien la referencia (Bedeutung) de una proposición (Satz) reside en un valor de verdad (Wahrheitswert) y no en una cosa existente o en un estado de cosas (Sachverhalt) (cf. la carta de Frege a Husserl del 24 de mayo de 1891, en Frege, G., Briefwechsel mit D. Hilbert, E. Husserl, B. Russell sowie ausgewählte Einzelbriefe, Hamburg, Meiner, 1980, p. 35).
} 
dar claro que la validez no debe derivarse de un modo de ser en el sentido de la realidad sensible -algo que sin dudas Platón suscribiría plenamente ${ }^{39}$. La validez (Geltung) tiene un valor en sí que tampoco debe entenderse en tanto 'modificación' del ser sensible (cf. Lotze 1874, p. 512). Es decir, nos encontramos aquí en las antípodas de todo tipo de naturalización de lógica. En otras palabras, el concepto de 'validez' tiene un sustento ontológico propio que no se deriva de otras formas de realidad óntica. Por dicho motivo, 'validez' no es un derivado sino que es un concepto fundamental (Grundbegriff): "también hay que considerar este concepto en tanto concepto fundamental que en todo sentido sólo se funda sobre sí mismo, que todo el mundo puede saber lo que se quiere decir con él [este concepto fundamental], pero que no podemos producir a través de una construcción a partir de partes (Bestandteilen), que no lo contuviesen ya al mismo" (Lotze 1874, p. 513).

En síntesis: la interpretación de la teoría de las ideas de Platón de Lotze consiste en afirmar que hay otros órdenes de realidad diferentes al ser de las cosas (sensibles); uno de ellos es el orden de la validez (Geltung). Es decir, puede haber verdades ('reales') aún cuando éstas no tengan un correlato sensible. En términos contemporáneos: su interés es la 'intensión', no la 'extensión', es decir, no le interesa saber si hay o no un objeto que corresponda a dichas verdades. Así, afirma que: "Platón no quería enseñar otra cosa (...) que la validez de las verdades (die Geltung der Wahrheiten), independientemente de que se confirmase en algún objeto del mundo exterior como siendo su especie ( $A r t)$; [no quería enseñar otra cosa que] el significado idéntico consigo mismo de las ideas, que siempre son lo que son, sin importar si hay cosas que a través de la participación en ellas aparecen en este mundo exterior, o si hay mentes (Geister) que, en la medida en que las piensan, les dan la realidad de un estado mental (Seelenzustandes) que acontece de sí. ${ }^{40}$ Pero al lenguaje griego de entonces y aún incluso después les faltó una expresión para este

\footnotetext{
${ }^{39}$ Lotze reconoce, incluso, que el error histórico en la interpretación de la teoría de las ideas de Platón consiste precisamente en interpretar las ideas a partir del ser de las cosas o de un modo similar (ähnlich) al ser de las cosas (cf. Lotze 1989b, p. 513). Este es un error habitual que se puede constatar en muchas aulas de filosofía, cuando se habla de dos 'mundos', como si se tratase de dos 'universos paralelos' situados en dos dimensiones topológicas distintas, pero análogos en su estructura ontológica. De ningún modo se debe hipostasiar esta dimensión de 'realidad' (cf. Lotze 1989b, p. 516), ya que al hacerlo se pierde el sentido profundo del platonismo (Platonischer Tiefsinn) (cf. p. 513). Por este motivo, Lotze recomienda tener mucho cuidado en el uso de conceptos filosóficos cuyo valor semántico es en muchos casos meramente analógico (como, por ejemplo, cuando hablamos de 'dos mundos'). Lotze atribuye incluso a Aristóteles buena parte del malentendido generado por su errónea interpretación de la teoría de las ideas de Platón (cf. p. 517).

40 En tal sentido, Lotze subraya que tampoco hay que malinterpretar a Platón en el sentido de creer que éste sostenía una suerte de dependencia de las ideas con relación al espíritu o sujeto que las piensa (cf. Lotze 1989b, p. 515). Las ideas son absolutamente independientes, aún del sujeto. En este pasaje, podríamos corroborar un gesto claramente 'anti-idealista' e incluso 'anti-intencionalista' por parte de Lotze.
} 
concepto de validez, que no encierra en sí ningún ser (der kein Sein einschließt)" (Lotze 1874, p. 513, el subrayado es mío; cf. p. 516).

La conclusión es entonces que el llamado 'mundo de las ideas' de Platón no es otro que el 'orden de la validez de las verdades' de Lotze, que tiene su modo de realidad propio (ihre eigne Weise der Wirklichkeit), que permanece inmutable a todo cambio y que tiene total independencia de todo tiempo (cf. Lotze 1874, p. 514). Con esto volvemos al punto de partida: el presunto devenir heraclíteo sólo se aplica al orden sensible, pero no al orden inmutable, ideal (platónico) de la validez de las verdades. Así llegamos una vez más a la conclusión de que el orden ontológico real de validez de las verdades es totalmente independiente del orden de realidad de lo óntico-sensible.

Cabe señalar que la validez se refiere en particular a las proposiciones verdaderas y sólo en un sentido derivado a sus partes, es decir, a los conceptos. En tal sentido, afirma Lotze: "sólo con una claridad parcial (mit halber Deutlichkeit) se puede transferir esta expresión [i.e., el valer (Gelten)] a conceptos individuales (einzelne Begriffe); de éstos sólo podemos decir que significan algo (etwas bedeuten); pero significan algo, porque a partir de ellos las proposiciones (Sätze) valen" (Lotze 1874 , p. 521). Si bien es cierto que aquí se marca una preponderancia de la proposición por sobre el concepto ${ }^{41}$, esto no debe entenderse necesariamente en sentido restrictivo, es decir, circunscribiendo la validez exclusivamente al terreno proposicional. Aún contando sólo con 'una claridad parcial', también se puede decir que los conceptos (en tanto unidades de sentido) entran en el orden de realidad de la 'validez'. Se puede interpretar esto en términos mereológicos, es decir, de 'partes' y 'todos': los conceptos serían así 'partes' de un 'todo' proposicional, y es a partir de ese 'todo' que las 'partes' tienen sentido. En una línea muy similar, Frege presentará luego su conocida idea del 'principio de contexto' (Kontext-Prinzip). 42 Por eso creemos que su hincapié en lo proposicional y en la verdad tiene que ver principalmente con su intención de brindar un fundamento sólido para las ciencias, en clara analogía con la posición de Frege. En un sentido similar a la teoría de la intuición categorial de Husserl, Lotze sostiene que es la intuición (Anschauung) la que produce la conexión entre representaciones (cf. Lotze 1874, p. 556), con lo cual vemos una vez más la importancia de entender esta relación (entre representaciones que conforman proposiciones) en términos de 'todos' y 'partes'. ${ }^{43}$ En cualquier caso,

\footnotetext{
41 Este punto es señalado correctamente por Alejandro Vigo en su excelente trabajo sobre la lógica de la validez y la fenomenología (cf. Vigo 2013, pp. 34-35).

42 El significado de las palabras (Bedeutung der Wörter) sólo se explica en conexión con la proposición (Satzzusammenhange) (cf. Frege 1884, p. xxii y $\S 60$ ). Aún así, los términos o palabras individuales (nombres propios y conceptos) no dejan de tener sus respectivos sentidos y referencias (cf. Frege, G., "Über Sinn und Bedeutung” (1892), en Frege, G., Funktion, Begriff, Bedeutung, Göttingen, Vandenhoeck \& Ruprecht, 1975).

43 Lamentablemente, no podemos analizar aquí la relación de Lotze con Frege y con Husserl.
} 
tanto las 'partes' como el 'todo' entran en el orden de la validez, es decir: 'validez' se predica no sólo de las proposiciones (el 'todo'), sino asimismo de los conceptos (las 'partes'); por otra parte, de ninguno de éstos puede decirse que 'son', puesto que el predicado 'ser' no corresponde a este tipo de realidad (cf. Lotze 1874, p. 569).

\section{Reflexiones finales}

A modo de conclusión, podemos corroborar, en primer lugar, que los tres autores analizados presentan una clara posición antipsicologista, que se manifiesta en su rechazo categórico a colocar la psicología o cualquier otra ciencia empírica en tanto fundamento de la lógica. En segundo lugar, vimos que la misma se sustenta sobre la base de un platonismo, en la medida que sostiene que hay unidades ideales de sentido que son independientes de la realidad sensible, de los actos mentales, y del lenguaje. Tanto Herbart como Lotze intentan demarcar explícitamente el sentido 'auténtico' del platonismo, de aquellas tradiciones que lo malinterpretaron al presentar las ideas en un mundo paralelo y análogo al sensible. En su rechazo a la hipóstasis o substanciación del orden ideal, vemos una coincidencia entre los tres autores; en ese sentido, podríamos decir que su platonismo lógico, semántico o de la validez no tiene un carácter metafísico, si por 'metafísico' entendemos la disciplina que estudia el 'ser'; la idealidad no se identifica con el ser de las cosas. En general, los tres pensadores coinciden al afirmar que se trata de un orden de realidad diferente al del ser de las cosas e independiente del mismo; este orden no es otro que el de las unidades ideales de sentido.

De los tres autores, Herbart es el que presenta una posición menos pulida, mientras que Bolzano y Lotze atienden a cuestiones más complejas. Si bien es cierto que Lotze presenta un platonismo más explícito (como también es el caso de Herbart), la posición de Bolzano es quizás la más compleja de las tres. ${ }^{44}$ Para Bolzano no sólo las proposiciones en sí tienen un valor semántico en sí, sino que además también lo tienen las representaciones en sí. Lotze -al igual que Frege algunos años despuéshace hincapié en las proposiciones, más que en las 'partes' (representaciones, conceptos) de las mismas, sin por eso, como vimos en el último capítulo, dejar de reconocer la validez de dichas 'partes'. A Lotze -al igual que a Frege y a diferencia de Bolzano- no le interesa el análisis de las proposiciones falsas, ya que su interés es dar un sustento 'real' a aquello que se dice o se predica como verdadero. En cualquier caso, podemos ver en todos ellos un punto coincidente en la idea de que hay

\footnotetext{
44 Para una lectura más detenida de Bolzano, cf. mi artículo: Niel, L., "Semántica y ontología. Reflexiones en torno a la Wissenschaftslehre de Bolzano", Revista Pensamiento, Universidad de Comillas, Serie Ciencia, Filosofía, Religión, Vol. 69, № 261, 2013.
} 
verdades en tanto tales ('en sí' diría Bolzano45), que son independientes de la existencia de un correlato cósico en el mundo sensible; aquí yace quizás la principal enseñanza de dichas autores.

Se podría reprochar que, de hecho, en el fondo de dicho platonismo subyace una especie de 'cosificación' ontológica de lo ideal, y que por eso se trata de una auténtica hipóstasis metafísica. ${ }^{46}$ Pero hemos podido ver en estas tres posiciones un claro intento de superar la metafísica cosificante, y que además muestran argumentativa y conceptualmente que en la base de todo pensar (sea éste una metafísica de tipo platónico, un idealismo absoluto, una posición psicologista, neurocientista, o incluso una filosofía del 'desocultamiento del ser') presuponemos siempre unidades de sentido ideales. Por supuesto, estamos haciendo alusión aquí básicamente a la lógica y a su validez, algo en lo que coincidirían los tres autores -y también Frege y Husserl ${ }^{47}$ - Bolzano va incluso más allá de lo lógico y de lo ontológico, llevando la dimensión del sentido (la semántica) a una dimensión propia que incluye incluso aquello que no es lógico ni ontológico, como por ejemplo las contradicciones 48 ; sin esta dimensión del sentido el pensamiento se disuelve en palabras vacías, dejando la puerta abierta a un relativismo que, por su parte, termina siendo un escepticismo que ni siquiera puede creer en sí mismo. Así, a partir de las tres posiciones presentadas, podemos observar -más allá de las diferencias- la apertura a una dimensión ideal del sentido 'en sí', que constituye la base misma de la lógica y en general de todo pensar posible. Podemos decir que se trata, malgré Heidegger, de un auténtico 'proto-fenómeno' (Urphänomen), sin el cual es difícil (o quizás incluso imposible) pensar en general la posibilidad de decir algo con sentido.

Más allá del valor histórico-filosófico del presente análisis, queremos formular aquí la siguiente pregunta: ¿por qué es importante plantear el tema del antipsicologismo y del platonismo en nuestra época? Cierto escepticismo metódico o deconstructivo no sólo es algo siempre bienvenido e incluso saludable, sino que además es

\footnotetext{
45 Lotze hace una referencia al mundo de las ideas como un "algo en sí y un algo que es siempre lo mismo" (ein an sich etwas und immer dasselbe Etwas) (Lotze 1874, p. 515, el subrayado es mío; cf. p. 556).

46 Esta es la posición que defiende Alejandro Vigo siguiendo la lectura de Heidegger, como él mismo reconoce explícitamente (cf. Vigo 2013, p. 37). Vigo retoma la lectura heideggeriana de Lotze, según la cual la 'validez' en tanto 'proto-fenómeno' (Urphänomen) o fenómeno originario termina siendo una "palabra mágica" (Zauberwort) o un "fetiche verbal" (Wortgötze) -los términos son de Heidegger, citados por Vigo (Vigo 2013, pp. 26-27, n.12). Dicha 'proto-fenomenalidad' presentaría así, según la lectura heideggeriana, una suerte de nec plus ultra que resolvería 'mágicamente' el problema del fundamento. Por supuesto, dicha interpretación se basa en los presupuestos de la filosofía heideggeriana del ser, que plantea el problema del 'ser' en términos no menos problemáticos que los de Lotze. Lamentablemente, no podemos abordar aquí estos temas.

47 En los Prolegómenos a las Logische Untersuchungen Husserl defiende la idea de que cualquier argumento presupone de base las leyes de la lógica, cf. Husserl 1900, p. 69.

48 Cf. supra, nota 44.
} 
fundamental a nivel metódico para someter nuestras creencias y convicciones a una auténtica evaluación crítica en aras de acercarnos a algún tipo de 'verdad' sobre la cual poder construir un discurso con sentido. El problema se presenta cuando dicho espíritu crítico se exacerba y adquiere el carácter de un relativismo escéptico, que pretende ser absolutamente radical y que eo ipso anula su propia validez y legitimidad. Incluso sin llegar a casos tan extremos, podemos constatar en nuestros días un cierto pulular generalizado de posiciones relativistas, sean ya psicologistas (e.g. neurocientistas), sociologistas, historicistas, etc. En este contexto, rescatar el sentido de una investigación auténticamente filosófica sobre la idealidad del sentido se presenta como una actividad necesaria en pos de captar ciertos órdenes de 'realidad' que no se dejan reducir a actos psicológicos, neurológicos, sociales o históricos (por mencionar sólo algunos).

En el presente artículo hemos pretendido presentar algunos aspectos centrales de la obra de tres referentes históricos del siglo XIX -casi olvidados-que sostienen un platonismo antipsicologista (¿y por qué no también antisociologista o antihistoricista o en general 'anti-reduccionista'?) fuerte. Más allá del aporte fundamental de sus argumentos, su relevancia radica además en el hecho de ser los antecedentes inmediatos de la primera fenomenología y de la primera filosofía analítica, quizás las dos corrientes filosóficas más importantes del siglo XX hasta nuestros días. En los tres casos, hemos sido testigos de un profundo compromiso con la filosofía y con la verdad a la que se puede llegar a partir de aquélla. Indudablemente, en los albores del siglo XXI no hay que caer en la candidez de creer que la verdad es algo 'a la mano', disponible y alcanzable de una sola vez en una suerte de 'metafísica de la presencia' dada o pre-dada. Pero la solución tampoco es negar dicha 'realidad' de la verdad, sino reconocer la inteligibilidad de esta dimensión de sentido, propia del análisis filosófico, que está allí para ser descubierta, escudriñada; dimensión de sentido que han intentado arrebatarla y reducirla, hasta incluso intentar negarla desde posiciones que van de un naturalismo a un sociologismo. El recorrido por la obra de Herbart, de Bolzano y de Lotze no ha tenido otro objetivo que mostrar la sutileza, precisión y profundidad conceptual de ciertos análisis filosóficos que, dentro de la convicción filosófica aquí sostenida, nos permite presentar una clara alternativa al reduccionismo (en particular psicologista), a partir de una base de sentido (platónica) que no se reduce ni a lo mental, ni a lo natural, ni a lo lingüístico, ni, a fortio$r i$, a lo social-histórico.

\section{Referencias bibliográficas}

Benoist, J. (1997), Phénoménologie, sémantique, ontologie. Husserl et la tradition logique autrichienne, Paris, PUF. 
Benoist, J. (2001), Représentations sans object. Aux origines de la phénoménologie et de la philosophie analytique, Paris, PUF.

Benoist, J. (2002), Entre acte et sens. La théorie phénoménologique de la signification, Paris, Vrin.

BEYER, C. (1996), Von Bolzano zu Husserl. Eine Untersuchung über den Ursprung der phänomenologischen Bedeutungslehre, Dordrecht, Kluwer.

Bolzano, B. (1837/1985)49, Wissenschaftslehre, Stuttgart / Bad Cannstatt, Friedrich Frommann Verlag.

Coffa, A. (1991), The Semantic Tradition from Kant to Carnap. To the Vienna Station, New York, Cambridge University Press.

Dummett, M. (1978), Truth and Other Enigmas, London, Duckworth.

Dummett, M. (1996), Frege and Other Philosophers, Oxford, Clarendon Press.

Frege, G. (1884/1990), Die Grundlagen der Arithmetik. Eine logischmathematische Untersuchung über den Begriff der Zahl, Hildesheim / Zürich, Georg Olms.

FREGE, G. (1893/1998), Grundgesetze der Arithmetik, Hildesheim / Zürich, Georg Olms.

Gabriel, G. (1989a), "Einleitung des Herausgebers. Lotze und die Entstehung der modernen Logik bei Frege", en Lotze 1989a.

Gabriel, G. (1989b), "Einleitung des Herausgebers. Objektivität: Logik und Erkenntnistheorie bei Lotze und Frege", en Lotze 1989b.

George, R. (2003), "Bolzano and the Problem of Psychologism", en Fisette, D., Husserl's Logical Investigations Reconsidered, Dordrecht, Kluwer, 2003, pp. 95-108.

Herbart, J. F. (1837/1993), Lehrbuch zur Einleitung in die Philosophie, Hamburg, Meiner.

Husserl, E. (1900/1975), Logische Untersuchungen. Erster Band. Prolegomena zur reinen Logik, Husserliana XVIII, The Hague, Nijhoff.

Husserl, E. (1979), Aufsätze und Rezensionen (1890-1910), Husserliana XXII, The Hague, Nijhoff.

JacQuette, D. (ed.) (2003), Philosophy, Psychology and Psychologism. Critical and Historical Readings on the Psychological Turn in Philosophy, Dordrecht, Kluwer.

Kusch, M. (1995), Psychologism. A Case Study in the Sociology of Philosophical Knowledge, London / New York, Routledge.

Lotze, R.H. (1874/1989a), Logik. Erstes Buch: Vom Denken, Hamburg, Meiner.

Lotze, R.H. (1874/1989b), Logik. Drittes Buch: Vom Erkennen, Hamburg, Meiner.

49 En el caso de referencias a textos fuentes se mencionará, en primer lugar, el año de edición original para tener una clara idea de la época en que la obra fue publicada. En segundo lugar, se indicará el año de la edición del texto utilizado para la realización del presente artículo. 
Morscher, E. (1972), "Von Bolzano zu Meinong: Zur Geschichte des logischen Realismus", en R. Haller (ed.), Jenseits von Sein und Nichtsein, Graz, Akademische Druck- und Verlagsanstalt, pp. 69-102.

OrTh, E.W. (1986), "Rudolf Hermann Lotze: Das ganze unseres Welt- und Selbstverständnisses", en Speck, J. (ed.), Grundproblem der großen Philosophen. Philosophie der Neuzeit IV, Göttigen,Vandenhoeck \& Ruprecht, pp. 9-51.

Sebestik, J. (1992), Logique et mathématique chez Bernard Bolzano, Paris, Vrin.

Simons, P. (1992), Philosophy and Logic in Central Europe from Bolzano to Tarski, Dordrecht, Kluwer.

Smith, B. (1996), Austrian Philosophy. The Legacy of Franz Brentano, Chicago, Open Court.

Stählin, L. (1889), Kant, Lotze and Ritschl. A Critical Examination, trad. Simon D.W., Edimburgh, T \& T Clark.

Vigo, A. (2013), Juicio, experiencia y verdad. De la lógica de la validez a la fenomenología, Navarra, Eunsa.

Luis Niel

CONICET / Universidad Nacional del Litoral, Argentina luisniel@yahoo.com 